\title{
Effect of polymerisation current density of electrodes on the performance of polypyrrole based redox-capacitor
}

\author{
W.A.D.S.S. Weerasinghe, K.P. Vidanapathirana*, K.S. Perera and C.M. Bandaranayake \\ Department of Electronics, Faculty of Applied Sciences, Wayamba University of Sri Lanka, Kuliyapitiya.
}

\begin{abstract}
Use of redox-capacitors is one of the effective and economic solutions to the demand for power sources. Redoxcapacitors replace the role of batteries and conventional capacitors by hundred times of the power density and energy density. Research activities are being continued to fabricate more comprehensive supercapacitors. Among those, fabrication of the symmetric polymer capacitor is dominant because of its simplicity. In this study, the effect of polymerisation current density on the performance of a symmetric polymer redoxcapacitor is reported. Conducting polymer, polypyrrole (PPy) was used as the electrodes, while a gel polymer electrolyte (GPE) based on polyvinylidenefluoride (PVDF) was used as the electrolyte. Fabrication of the redox-capacitors was carried out in the configuration of PPy : GPE : PPy. They were characterised using cyclic voltammetry tests, continuous galvanostatic charge-discharge tests and electrochemical impedance spectroscopy. An average specific capacitance value of $12 \mathrm{Fg}^{-1}$ could be obtained by cyclic voltammetry measurements. It was about $7 \mathrm{Fg}^{-1}$ as per the galvanostatic charge-discharge test. The specific capacity remains rather stable upon continuous cycling. An increase was noticed in specific capacity with the increase in polymerisation current density. From the impedance spectroscopy measurements, it was observed that with increasing polymerisation current density, the capacitive nature of the redox-capacitors can be improved.
\end{abstract}

Keywords: Cyclic voltammetry, polypyrrole, redox-capacitor, specific capacity.

\section{INTRODUCTION}

A supercapacitor or an electrochemical capacitor is an energy storage device, which is used to store and release energy (Uppugalla et al., 2014). By bridging the gap between batteries and conventional capacitors, supercapacitors have been designed for higher energy storing and elevated power delivery (Snook et al., 2011). Having both technical and economical advantages in a diverse range of consumer and industrial applications including hybrid electric vehicles, potable electronics, medical electronics and military devices, supercapacitors combine the high energy storage capability of batteries with the high power delivery capability of capacitors (Fonseka et al., 2006).

Supercapacitors have been categorised into two general classes on the type of electrode materials employed and the charge storage mechanism at the electrode-electrolyte interfaces (Halper \& Ellenbogen, 2006; Hashmi et al., 2007) as electrochemical double layer capacitors (EDLCs), and redox-capacitors. EDLCs store charges electrostatically (non-faradically), which govern the double layer capacitance where there is no transfer of charge between the electrode and electrolyte. It utilises an electrochemical double layer of charge to store energy. Different forms of carbon materials such as activated carbon, carbon aerogels and carbon nanotubes are employed in the formation of electrodes (Frackowiak \& Beguin, 2001; Prabaharan et al., 2006; Gu \& Yushin, 2013). In contrast to the EDLCs, redox-capacitors store charge faradically through the transfer of charge between the electrode and electrolyte. This is accomplished through electrosorption, reduction-oxidation reactions and intercalation processes. These faradic processes allow redox-capacitors to achieve greater capacitance and energy densities than EDLCs (Halper \& Ellenbogen, 2006). Transition metal oxides such as $\mathrm{RuO}_{\mathrm{x}}, \mathrm{CoO}$, and $\mathrm{NiO}_{x}$ or conducting polymers such as polypyrrole (PPy), 
polyaniline (PANI) and polythiophene (PT) can be used as electrode materials for redox-capacitors. Conducting polymers have been regarded as promising capacitive materials because the ion uptake during doping allows ion intercalation throughout the electrode and exposes the ions in the electrolyte to more than just the surface interface of the material (Yu et al., 2013). The use of conducting polymer materials for redox capacitors has several advantages over other systems. They have a good intrinsic conductivity and are relatively cheap, so the preparation and fabrication costs would be competitive. Among conducting polymers PPy has attracted much attention as a better candidate due to its ease of synthesis, good redox properties, stability in oxidised form, the ability to give high electrical conductivity, water solubility, commercial availability and useful electrical and optical properties (Ates et al., 2012).

Redox-capacitors have been fabricated using liquid electrolyte as the separator. However, liquid electrolytes pose crucial problems such as self-discharge, corrosion, leakage and low energy density (Jayathilaka et al., 2014). To overcome these problems, the trend has been the utilisation of gel polymer electrolytes (GPE). The fabrication of a redox-capacitor based on a GPE and a conducting polymer PPy as electrodes, and the variation of specific capacitance with respect to the current density used for the synthesis of polymer electrodes has been the focus of this study.

\section{METHODOLOGY}

\section{Preparation of the electrode}

The monomer pyrrole (Aldrich) was distilled and stored under refrigeration prior to use. Pyrrole was galvanostatically electro-polymerised on to fluorine doped tin oxide (FTO) glass using Metrohom-Autolab 101 Potentiostat/Galvanostat. For this, a 3-electrode set up was used where $\mathrm{Ag} / \mathrm{AgCl}$ and $\mathrm{Pt}$ electrodes served as reference and counter electrodes, respectively. The monomer concentration (pyrrol) was $0.1 \mathrm{M}$. Sodium dodecylbenzenesulfonate (SDBS) (Aldrich) of concentration $0.05 \mathrm{M}$ was used as the salt. Thickness of the PPy film was maintained at $1 \mu \mathrm{m}$. PPy electrodes were prepared with different current densities from $0.5-2.0 \mathrm{~mA} \mathrm{~cm}^{-2}$.

\section{Preparation of GPE}

GPE was prepared using polyvinylidenefluoride (PVdF) (Aldrich), zinc trifluoromethanesulfonate $\left(\mathrm{Zn}\left(\mathrm{CF}_{3} \mathrm{SO}_{3}\right)_{2}\right.$ - ZnTF) (Aldrich), ethylene carbonate (EC) (Aldrich) and propylene carbonate (PC) (Aldrich) as previously reported by Jayathilake et al. (2014).

\section{Fabrication of redox-capacitors}

Symmetric redox-capacitors were fabricated using two PPy films deposited on FTO glass pieces having $1 \mathrm{~cm}^{2}$ electrode area. GPE having the same area was used as the electrolyte. Structure of the redox-capacitor was in the form of PPy:DBS / PVdF:EC:PC:ZnTF / PPy:DBS.

\section{Characterisation}

Electrochemical impedance spectroscopy (EIS) measurements were carried out at frequencies ranging from $0.01 \mathrm{~Hz}-400 \mathrm{kHz}$ using Metrohm AUTOLAB M101 frequency response analyser.

Cyclic voltammetry tests were performed in a potential window of $(-0.4)-0.5 \mathrm{~V}$ at a scan rate of 10 $\mathrm{mV} / \mathrm{s}$ by means of a computer controlled Potentiostat/ Galvanostat (Metrohom- AUTOLAB M101). One PPy electrode served as the working electrode and the other as both the counter and reference electrodes. Continuous galvanostatic charge-discharge tests were carried out to check the ability to withstand long term cycling. The redox-capacitor was first galvanostatically charged to $0.5 \mathrm{~V}$, and immediately subjected to a galvanostatic discharge of $0.0 \mathrm{~V}$. The maximum charge and discharge currents were set to $25 \mu \mathrm{A}$. The specific capacitance of the redox-capacitor was calculated using the chargedischarge curves.

\section{RESULTS AND DISCUSSION}

The impedance plots obtained for the redox-capacitors fabricated using PPy electrodes having different polymerisation current densities are shown in Figure 1. The ideal impedance behaviour of a pure capacitor is a straight line parallel to the imaginary axis (Z") whereas in practical capacitors, a steep rising capacitive impedance response is observed in the low frequency region (Panday et al., 2010). In the resulting plots, slanted straight lines were observed in the lower frequency range. This exhibits the capacitive behaviour of the redox-capacitors. The important feature is the tendency of the lines to become more parallel with the imaginary axis when the polymerisation current density increases. Then, it can be elucidated that the capacitive behaviour of the redoxcapacitors enhances at high current densities.

Cyclic voltammograms obtained for the fabricated redox-capacitors are shown in Figure 2. 


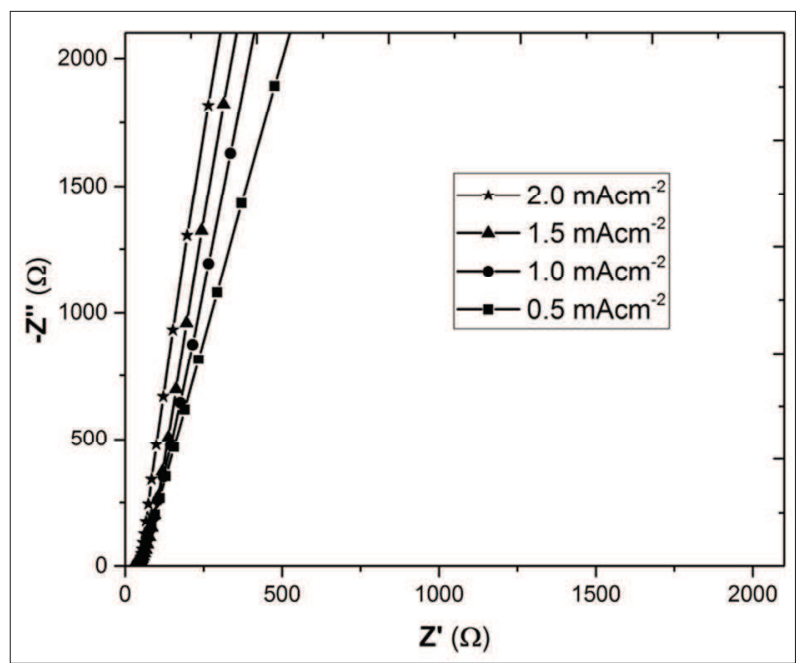

Figure 1: Impedance plots for the redox-capacitors in the frequency range of $0.01 \mathrm{~Hz}$ to $400 \mathrm{kHz}$ at room temperature

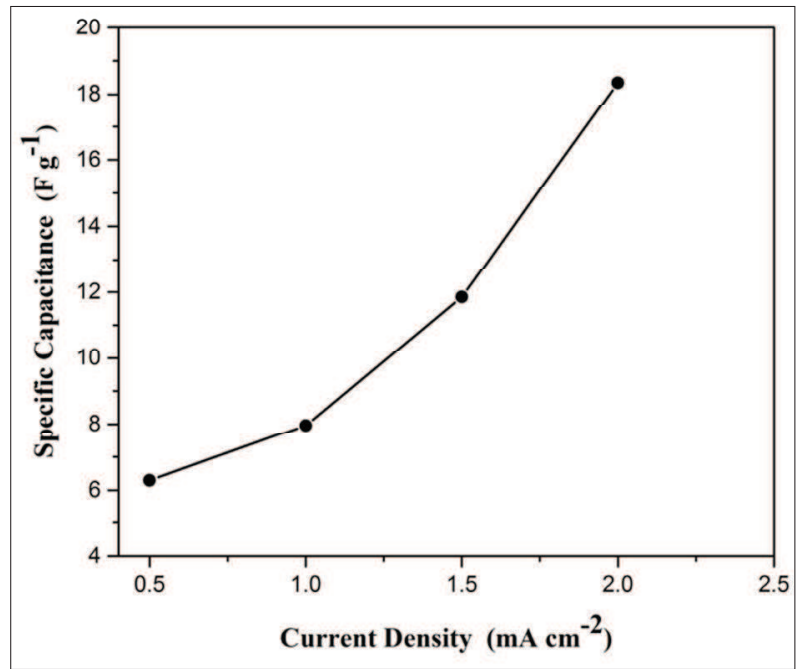

Figure 3: Variation of specific capacitance of redox-capacitors with polymerisation current density of PPy electrodes

The rectangular shape of the cyclic voltammograms indicates the capacitive nature of the redox-capacitors (Dey et al., 2015). In addition, this points to a fast switching rate of ions at the sites of electrode-electrolyte interfaces. Featureless characteristic behaviour of the obtained cyclic voltammograms is due to the occurrence of electron transfer between the electrode and the gel electrolyte at a constant rate (Hashmi et al., 2007). Cyclic voltammograms obtained during charging-discharging processes of redox-capacitors showed almost symmetric current responses. This may be due the fact that the fabricated redox-capacitors have a good electrochemical

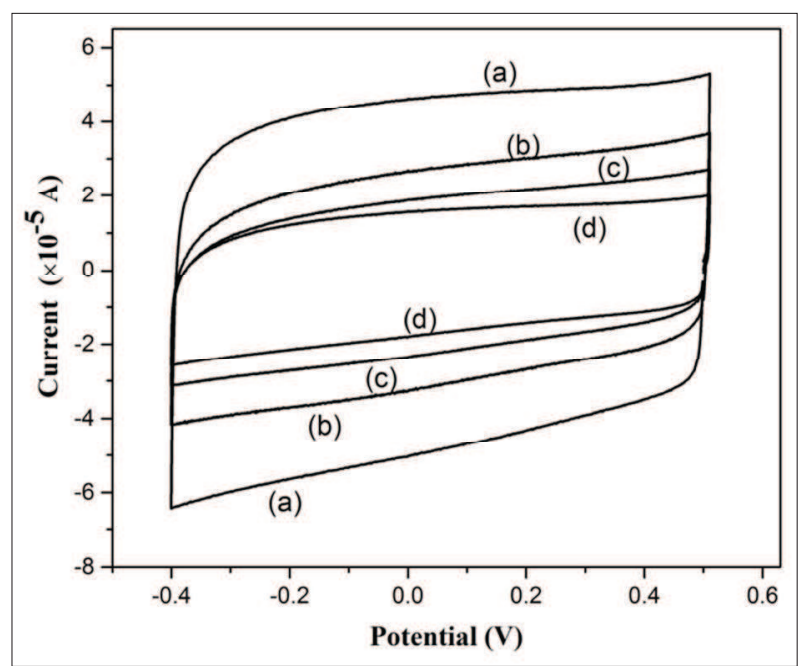

Figure 2: Cyclic voltammograms obtained with the scan rate of $10 \mathrm{mV} \mathrm{s}^{-1}$ for the redox-capacitors with PPy films polymerised at different current densities. (a) $2.00 \mathrm{~mA} \mathrm{~cm}^{-2}$; (b) $1.50 \mathrm{~mA} \mathrm{~cm}^{-2}$; (c) $1.00 \mathrm{~mA} \mathrm{~cm}{ }^{-2}$; (d) $0.50 \mathrm{~mA} \mathrm{~cm}^{-2}$

reversibility within the potential window used (Wu et al., 2010).

The specific capacitance $\left(\mathrm{C}_{\mathrm{s}}\right)$ values were calculated using the following equation (Wang et al., 2013).

$$
\mathrm{C}_{\mathrm{s}}=\left(2 \int \mathrm{I} . \mathrm{dV}\right) / \mathrm{m} . \Delta \mathrm{V} . \mathrm{S}
$$

where $\int \mathrm{I} . \mathrm{dV}$ is the integrated area of the CV curve, $\mathrm{m}$ is the single electrode mass, $\Delta \mathrm{V}$ is the potential window and $\mathrm{S}$ is the scan rate.

The expanding nature of cyclic voltammograms with increasing polymerisation current density shows an increase in specific capacity of the redox-capacitors. This is shown in Figure 3.

As the thickness of the PPy film was kept at $1 \mu \mathrm{m}$ and the same electrolyte was used for all redox-capacitors, the increase in specific capacitance with polymerisation current density might be due to the increase in the migration rate of ions from the electrolyte into the polymer electrode (intercalation of $\mathrm{Zn}^{+}$) for faradic reactions. It is reported in literature that by increasing the polymerisation current density, the porosity of the PPy:DBS electrode is enhanced (Zheng, 2011). The increase of porosity of the electrode material structure supports better intercalation processes, as porosity makes the access of ions to the structure of PPy film easier. This results in an increase of specific capacity. 


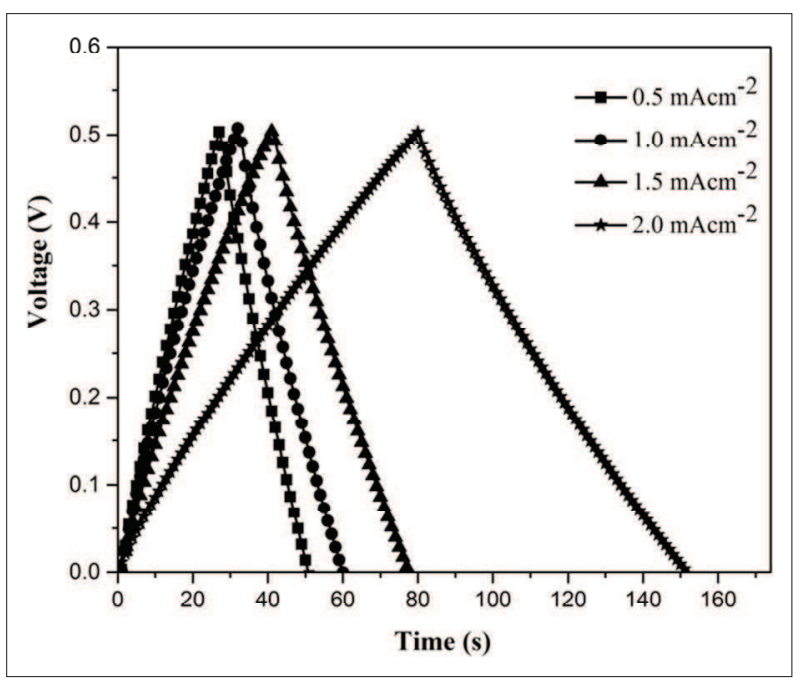

Figure 4: Charging discharging curves of the redox-capacitors with the variation of PPy polymerisation current density

Figure 4 shows the charge-discharge characteristics of the redox-capacitors at constant current. The discharge characteristics are found to be almost linear and symmetric, which symbolise a good capacitive behaviour. Specific capacitance values were calculated from the linear part of the discharge curves using the relationship $\mathrm{C}_{\mathrm{s}}=2 i / \mathrm{m}(\mathrm{dv} / \mathrm{dt})$, where $\mathrm{m}$ is the mass of PPy electrode, $i$ is the discharge current and $\mathrm{dv} / \mathrm{dt}$ is the slope of the discharge curve (Wang et al., 2013). The calculated specific capacitance values varied from 3.97 to $11.94 \mathrm{Fg}^{-1}$ when the polymerisation current density varied from 0.5 to $2.0 \mathrm{~mA} \mathrm{~cm}^{-2}$, respectively.

Figure 5 shows the variation of specific capacitance of the redox-capacitors with cycle number for four capacitors having PPy electrodes polymerised at different current densities. Initially, the capacitance value has increased upon cycling. This may be the result of irreversible charge consumption due to some reactions associated with possible oxidation and reduction of loosely bound surface groups at the electrode-electrolyte interface. It is also evident that the specific capacitance values are maintained at a steady level after sometime. This is one of the most important and special features of the redox-capacitors when compared to rechargeable cells (Hashmi et al., 2005). It is also clear that the specific capacitance value increases with the increase of current density as observed with cyclic voltammetry.

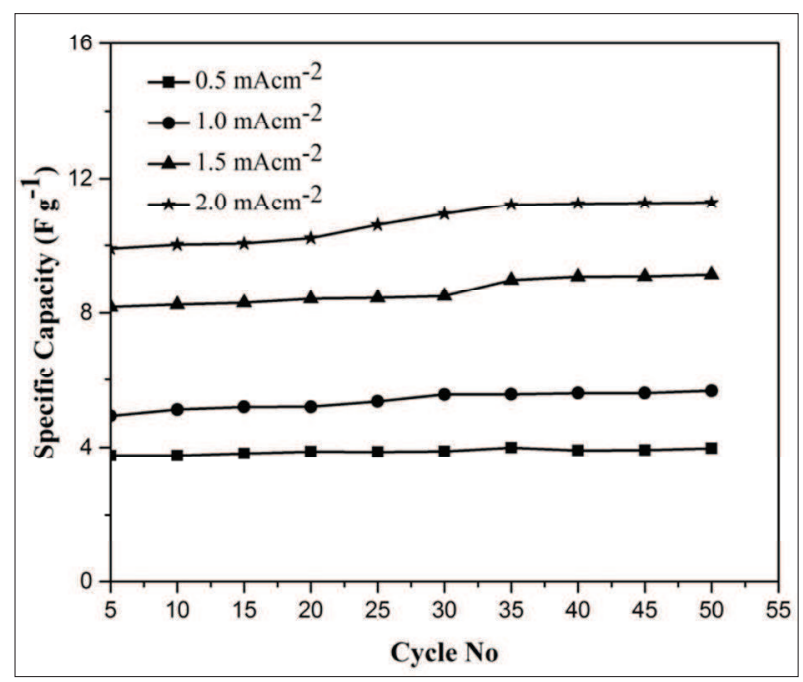

Figure 5: Variation of specific capacitance of the redox-capacitors with the cycle number calculated from galvanostatic charge-discharge tests

\section{CONCLUSION}

The electrochemical properties of redox-capacitors structured as PPy:DBS/PVdF:EC:PC:ZnTF/PPy:DBS were investigated by varying the polymerisation current density of electrodes utilising impedance spectroscopy, cyclic voltammetry and galvanostatic charge-discharge techniques. From the impedance spectroscopy measurements, it was noted that with increasing polymerisation current density, the capacitive nature of the redox-capacitors can be improved. An average specific capacitance value of $12 \mathrm{Fg}^{-1}$ could be obtained by cyclic voltammetry measurements. It was about $7 \mathrm{Fg}^{-1}$ as per the galvanostatic charge-discharge test. The specific capacity remains rather stable upon continuous cycling. An increase in the specific capacity was noticed by increasing the polymerisation current density from both cyclic voltammetry and continuous charge-discharge tests. Although the values were little lower, it may be improved by enhancing the quality of the GPE and varying the thickness of the electrodes.

\section{Acknowledgement}

The National Science Foundation of Sri Lanka (Grant No: RG/2014/BS/01) and the Wayamba University of Sri Lanka (Grant No: SRHDC/RP/04/14/01) are gratefully acknowledged for the financial support. 


\section{REFERENCES}

1. Ates M., Karezehir T. \& Sarac A.S. (2012). Conducting polymer and their applications. Current Physical Chemistry 2(3): $224-240$.

DOI: https://doi.org/10.2174/1877946811202030224

2. Dey R.S., Hjuler H.A. \& Chi Q. (2015). Approaching the theoretical capacitance of graphene through copper foam integrated three dimensional graphene networks. Journal of Materials Chemistry A3(12): 6225 - 6689.

DOI: https://doi.org/10.1039/C5TA01112D

3. Fonseka C.P., Joao E. \& Neves S. (2006). Poly(3methil thiophene)/PVDF composite as an electrode for supercapacitors. Journal of Power Sources 158: 789 - 794. DOI: https://doi.org/10.1016/j.jpowsour.2005.08.050

4. Frackowiak E. \& Beguin F. (2001). Carbon materials for the electrochemical storage of energy in capacitors. Carbon 39: $937-950$.

5. Gu W. \& Yushin G. (2013). Review of nanostructured carbon materials for electrochemical capacitor applications: advantages and limitations of activated carbon, carbidederived carbon, zeolite-templated carbon, carbon aerogels, carbon nanotubes, onion-like carbon, and grapheme. Wiley Inter Disciplinary Reviews: Energy and Environment 3/5: $424-474$.

6. Halper S.M. \& Ellenbogen J.C. (2006). Supercapacitors: A Brief Overview, pp. 1 - 34. MITRE Nanosystems Group, MITRE Corporation, McLean, Virginia, USA.

7. Hashmi S.A., Kumar A. \& Tripahi S.K. (2005). Investigations on electrochemical supercapacitors using polypyrrole redox electrodes and PMMA based gel electrolytes. European Polymer Journal 41: 1373 - 1379. DOI: https://doi.org/10.1016/j.eurpolymj.2004.12.013

8. Hashmi S.A., Kumar A. \& Tripathi S.K. (2007). Experimental studies on poly methyl methacrylate based gel polymer electrolyte for application in electrical double layer capacitors. Journal of Physics D: Applied Physics 40: $6527-6534$.
DOI: https://doi.org/10.1088/0022-3727/40/21/010

9. Jayathilake Y.M.C.D., Perera K.S., Vidanapathirana K.P. \& Bandara L.R.A.K. (2014). A novel gel polymer electrolyte based on polymethylmethacrylate and copper trifluoromethanesulfonate. Journal of Electroanalytical Chemistry 724: 125 - 129 .

DOI: https://doi.org/10.1016/j.jelechem.2014.04.018

10. Pandey G.P., Kumar Y. \& Hashmi S.A. (2010). Ionic liquid incorporated polymer electrolyte for supercapacitor application. Indian Journal of Chemistry 49A: 743 - 751.

11. Prabaharan S.R.S., Vimala R. \& Zanial Z. (2006). Nanostructured mesoporous carbon electrodes for supercapacitors. Journal of Power Sources 161: 730 - 736. DOI: https://doi.org/10.1016/j.jpowsour.2006.03.074

12. Snook G.A., Kao P. \& Best A.S. (2011). Conductingpolymer-based supercapacitor devices and electrodes. Journal of Power Sources 196: $01-12$.

13. Uppugalla S., Male U. \& Srinivasan P. (2014). Design and synthesis of heteroatoms doped carbon/polyaniline hybrid material for high performance electrode in supercapacitor application. Electrochimica Acta 146: 242 - 248.

DOI: https://doi.org/10.1016/j.electacta.2014.09.047

14. Wang W., Guo S., Penchev M., Ruiz I., Bozhilov K.N., Yan D., Ozkan M. \& Ozkan C.S. (2013). Three dimensional few layer grapheme and carbon naotube foam architectures for high fidelity supercapacitors. Nano Energy 2: 294 - 303. DOI: https://doi.org/10.1016/j.nanoen.2012.10.001

15. Wu Z.S., Ren W., Gao L., Liu B., Pei S. \& Cheng H.M. (2010). High-energy $\mathrm{MnO}_{2}$ nanowire/graphene and graphene asymmetric electrochemical capacitors. ACS Nano 4/10: 5835 - 5842.

DOI: https://doi.org/10.1021/nn101754k

16. Yu A., Chabot V. \& Zhang J. (2013). Electrochemical Supercapacitors for Energy Storage and Delivery: Fundamental and Applications, $1^{\text {st }}$ edition, pp.165 - 167. CRC press, Boca Raton, USA.

17. Zheng W. (2011). Analysis of high performance polypyrrole actuators. PhD thesis, University of Wollongong, New Zealand. 